\section{Atenção aos defeitos congênitos no Brasil: panorama atual}

\author{
Birth defects and health strategies in Brazil: \\ an overview
}

\author{
1 Instituto Fernandes \\ Figueira, Fundação Oswaldo \\ Cruz, Rio de Janeiro, Brasil. \\ 2 Instituto de Medicina \\ Social, Universidade \\ do Estado do Rio de Janeiro, \\ Rio de Janeiro, Brasil. \\ Correspondência \\ D. D. G. Horovitz \\ Centro de Genética Médica \\ José Carlos Cabral \\ de Almeida, Instituto \\ Fernandes Figueira, \\ Fundação Oswaldo Cruz. \\ Av. Ruy Barbosa 716, \\ Rio de Janeiro, RJ \\ 22250-020, Brasil. \\ dafne@ceresgenetica.com.br
}

\begin{abstract}
Birth defects have increased progressively in Brazil, shifting from the fifth to the second cause of infant mortality from 1980 to 2000, thus highlighting the need for specific health policy strategies. Some governmental and nongovernmental actions related to birth defects in Brazil include information services on teratogenic agents and inborn errors of metabolism, monitoring of birth defects, neonatal screening and treatment of some genetic diseases, and rubella immunization. In addition, flour fortification with folic acid for prevention of certain birth defects has begun recently. Despite the importance of such initiatives, it is still difficult to view birth defects from a comprehensive perspective. A specific national policy on birth defects must be formulated. Active participation is needed by the Ministry of Health, using existing genetic services as the backbone, in order to develop a regionalized, hierarchical, and functional network related to birth defects in Brazil.

Birth Defects; Health Policy; Genetic Services; Medical Genetics; Health Planning
\end{abstract}

Dafne Dain Gandelman Horovitz 1 Juan Clinton Llerena Jr. 1 Ruben Araújo de Mattos 2

\section{Introdução}

Os defeitos congênitos vêm apresentando relevância crescente como causa de sofrimento e prejuízos à saúde da população. Define-se como malformação congênita a anomalia estrutural presente ao nascimento 1 . Uma definição mais ampla seria a expressão "defeito congênito" (tradução do inglês "birth defect"), incluindo toda anomalia funcional ou estrutural do desenvolvimento do feto decorrente de fator originado antes do nascimento, seja genético, ambiental ou desconhecido, mesmo quando o defeito não for aparente no recém-nascido e só manifestar-se mais tarde 2 .

Estudos indicam que a incidência geral dos defeitos congênitos na América Latina não difere, significativamente, daquela encontrada em outras regiões do mundo ${ }^{3}$. De modo geral, pode-se considerar que não menos de 5,0\% dos nascidos vivos apresentam alguma anomalia do desenvolvimento, determinada, total ou parcialmente, por fatores genéticos. Acrescentando-se os distúrbios que se manifestam mais tarde, como certas enfermidades crônicas degenerativas, é ainda mais evidente o considerável efeito que têm os fatores genéticos sobre a saúde 2 .

Apesar dos fatores genéticos estarem implicados em praticamente todas as doenças, como resultado de sua interação com o ambiente, o papel relativo do componente genético 
poderá ser maior ou menor 4 . Dentre as doenças nas quais o componente genético é preponderante, podem ser citadas as monogênicas, individualmente raras, porém acometendo como grupo $2,0 \%$ da população geral, as cromossômicas, presentes em $0,7 \%$ dos nascidos vivos (e em metade dos abortamentos espontâneos) e as multifatoriais, responsáveis por grande parte das malformações congênitas e também por muitos problemas comuns da vida adulta 4 . Neste artigo, em alguns momentos, os termos "defeito congênito" e "doença genética” são usados como sinônimos, apesar de, atualmente, praticamente todas as doenças serem "genéticas" em algum aspecto (mesmo uma eventual predisposição para doença infecciosa). Na realidade, tais termos, no âmbito deste texto, referem-se às doenças monogênicas, cromossômicas e às malformações congênitas, nas quais o papel da genética clínica (também utilizada como sinônimo de genética médica) é de reconhecida importância. No Brasil, a prática médica nessa área teve início recente quando comparada com outras especialidades.

Diante do exposto sobre os defeitos congênitos, tivemos como objetivo determinar a relevância desses no Brasil, mapeando estratégias já implementadas para sua abordagem. Apenas após a obtenção de tais dados, caso definido que se trata de problema significativo, poderá ser estruturada política pública na área.

Foi realizada uma análise documental, tomando como fonte documentos oficiais emitidos pelo Ministério da Saúde (MS), notadamente portarias ministeriais e da Secretaria da Assistência à Saúde, resoluções de agências governamentais e manuais operacionais do $\mathrm{Mi}$ nistério. A análise dessas fontes foi executada tendo como guia as proposições da análise temática 5. Paralelamente, a partir de um instrumento de coleta previamente elaborado e validado, lançou-se mão dos seguintes bancos de dados disponibilizados pelo MS: Sistema de Informações sobre Mortalidade (http:/ / tabnet.datasus.gov.br/cgi/sim/obtmap.htm, acessado em 31/Out/2003) e Sistema de Informações Hospitalares (http://tabnet.datasus.gov.br/tabnet/ tabnet.htm\#Morbidade, acessado em 31/Out/ 2003). Procurou-se basicamente identificar: (a) evolução da mortalidade proporcional por causas em menores de um ano entre 1980 e 2000; (b) permanência hospitalar de pacientes entre 0 a 19 anos; (c) mortalidade hospitalar para esse mesmo grupo etário e; (d) correlações desses dados com o capítulo XVII da Classificação Internacional de Doenças (CID) 6 ("malformações congênitas, deformidades e anomalias cromossômicas").
Finalmente, com o objetivo de localizar outras ações relacionadas aos defeitos congênitos no Brasil, foram utilizadas informações obtidas através de um questionário fechado enviado aos serviços de genética médica brasileiros 7 , notadamente aquelas relacionadas a linhas de pesquisa/projetos extramurais. Foi confeccionada planilha no programa Excel, sendo tabuladas todas as informações colhidas de modo a ter-se um mapa das principais características dos serviços, em especial, quanto a: (a) oferta, (b) distribuição geográfica, (c) financiamento, (d) características do atendimento, (e) linhas de pesquisa e (f) projetos extramurais. Uma vez sinalizada a existência de atendimentos específicos (diferenciados da rotina de atendimento clínico dos serviços de genética), solicitou-se informação adicional sobre os mesmos. Os dados assim coletados foram analisados inter e intra fontes e depois cruzados com a produção atual de literatura sobre defeitos congênitos e natureza dos serviços a eles dirigidos.

Para melhor expor e discutir os resultados obtidos, subdividiu-se o presente artigo em três seções. A primeira aborda o crescente impacto dos defeitos congênitos na saúde, ilustrados pelos levantamentos da evolução das causas proporcionais de mortalidade e do peso dos defeitos congênitos na morbidade hospitalar no Brasil. A segunda seção mostra as ações disponíveis no país relacionadas aos defeitos congênitos (governamentais e não-governamentais); tais ações incluem monitorização dos defeitos congênitos, informações sobre teratógenos e erros metabólicos, triagem neonatal, tratamento de doenças genéticas e medidas preventivas para defeitos congênitos específicos. Finalmente, na última seção, são tecidas considerações acerca das informações obtidas e, apresentadas propostas para elaboração de política de saúde voltada para os defeitos congênitos.

\section{Defeitos congênitos e seu impacto na saúde}

A mortalidade infantil é um importante indicador de saúde de um país ou comunidade, por estar associado a fatores como saúde materna, qualidade e acesso a serviços de saúde, condições sócio-econômicas e práticas de saúde pública. Quando estratificadas as causas de mortalidade infantil, observa-se, em várias regiões do mundo, uma diminuição na taxa total de óbitos no grupo, em especial, nas causas infecciosas; como resultante, a proporção de mortes atribuíveis às malformações congênitas vem 
aumentando 8,9. Outro dado relacionado às anomalias congênitas, além da mortalidade, é a maior morbidade, definida como risco para o desenvolvimento de complicações clínicas, incluindo número de internações e gravidade das intercorrências. A maioria das doenças nãoinfecciosas, maior causa dos óbitos em nações desenvolvidas, provavelmente têm um componente genético 10,11 .

Também, no Brasil, em hospitais de referência, as malformações congênitas assumem papel importante na morbidade e mortalidade. No Instituto Fernandes Figueira (IFF), Fundação Oswaldo Cruz (FIOCRUZ), Rio de Janeiro, de janeiro de 1999 a julho de 2003, malformação congênita foi um dos três diagnósticos principais codificados na internação em $37,0 \%$ das admissões hospitalares pediátricas. A mortalidade hospitalar, no grupo com malformação, foi $9,8 \%$, o dobro daquela do grupo sem malformação 12. Em 1986, 13,4\% de um grupo de pacientes acompanhados pelo serviço de genética da Fundação Faculdade Federal de Ciências Médicas de Porto Alegre, Rio Grande do Sul, faleceram, numa proporção quase cinco vezes superior ao grupo controle, sem defeitos congênitos 13. Dado semelhante foi observado na cidade de São Paulo, com uma mortalidade quatro vezes maior num grupo com anomalias congênitas quando pareado com controles sem malformação 14. Além da gravidade, foram dobrados o tempo e o custo das internações dos pacientes com defeitos congênitos em relação aos controles 13 .

Ao levantar, na base de dados do Departamento de Informática do SUS (DATASUS - http: // tabnet.datasus.gov.br/tabnet/tabnet . htm\#Morbidade), as informações relacionadas a malformações congênitas, observa-se que a mortalidade hospitalar, em 2002, na faixa etária pediátrica (0 a 19 anos), foi de 1,13\%, passando para $3,33 \%$ quando o capítulo CID do diagnóstico era o XVII ("malformações congênitas, deformidades e anomalias cromossômicas"). Na faixa de menores de um ano, a mortalidade hospitalar foi $3,59 \%$, sendo $9,99 \% \mathrm{em}$ casos com malformação. Ainda neste levantamento, a permanência hospitalar média, em caso de malformação, foi de 5,7 dias, contra uma média de 4,5 dias. No quesito custo, o dado é ainda mais significativo, com valor estimado em $\mathrm{R} \$ 1.168,93$ nas internações com malformação congênita, contra um valor médio das AIH (Autorização de Internação Hospitalar) de $\mathrm{R} \$ 365,74$.

Além da alta morbi-mortalidade, outra problemática envolvida em tais patologias é a cronicidade. O indivíduo com doença crônica ne- cessita de tratamento contínuo, implicando em altos custos. A estimativa de custo-vida médio por criança para as anomalias congênitas deve compreender, além do tratamento médico, serviços do desenvolvimento (como fisioterapia, fonoaudiologia, terapia ocupacional), educação especial ou inclusiva, perda da produtividade por incapacidade ou morte e perda na arrecadação salarial familiar do responsável pelos cuidados da criança 15. A esse cálculo deveriam também ser adicionados os custos psicossociais, como trauma psicológico da família e dificuldades de adaptação à sociedade "normal”, inclusive com grande risco de desestruturação familiar.

Através dos dados computados pelo MS, no banco de dados do Sistema de Informações sobre Mortalidade, pode ser observada a evolução das causas de mortalidade infantil desde 1980. A Figura 1 apresenta graficamente a evolução da mortalidade proporcional entre os menores de um ano nos cinco principais grupos de causa de óbito entre os anos de 1980 e 2000 no Brasil.

Quando excluídas e redistribuídas proporcionalmente as causas mal definidas, as causas perinatais eram a principal causa de mortalidade infantil no Brasil em 1980, responsáveis por $38,0 \%$ dos óbitos em menores de um ano, ocupando as anomalias congênitas a quinta posição, com 5,0\% do total. Em 1990, o perfil começou a mudar, notando-se uma redução proporcional nas causas infecciosas e nutricionais, passando as anomalias congênitas para $8,0 \%$ e quarta causa. No último ano avaliado (2000), as diferenças foram bastante significativas, com grande redução proporcional dos óbitos por causas infecciosas e respiratórias, que passaram para menos de $10,0 \%$, assumindo as malformações congênitas a segunda posição, como causa de 13,0\% dos óbitos em menores de um ano. Essas estatísticas referentes aos defeitos congênitos e mortalidade infantil no Brasil podem ser classificadas como surpreendentes, considerando sua magnitude e a total ausência de políticas governamentais relacionadas à prevenção e ao manejo desse grupo de problemas.

\section{Ações já existentes no Brasil relacionadas aos defeitos congênitos}

A seguir, serão descritos alguns programas especiais relacionados aos defeitos congênitos. Trata-se, na maioria, de iniciativas isoladas, algumas organizadas por grupos técnicos ou serviços de genética, preocupados com questões 
Figura 1

Evolução do número de óbitos por grupo de causa de mortalidade em menores de um ano de 1980 a 2000 no Brasil.

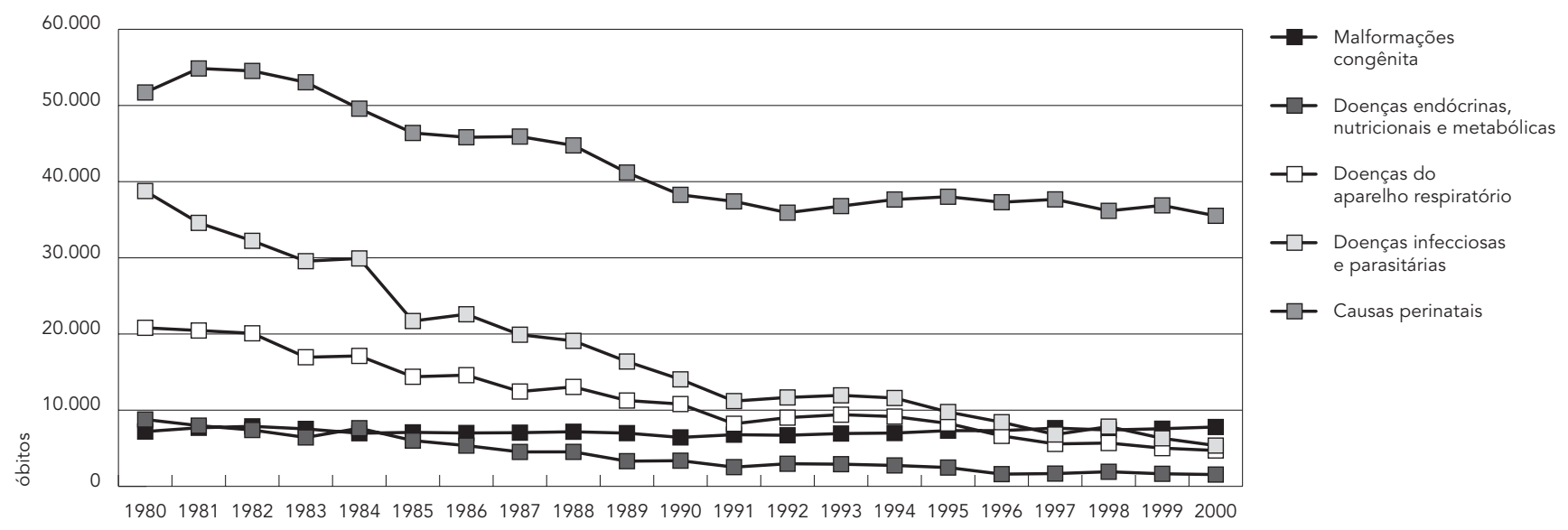

Fonte: Sistema de Informações sobre Mortalidade, acessado através do DATASUS (http://www.datasus.gov.br).

pontuais; outras, muitas vezes, oriundas de pressões exercidas por grupos de pacientes, demandando diagnóstico ou tratamento, refletindo a ausência do Estado nas formulações. Tais programas sinalizam uma tímida apresentação da questão dos defeitos congênitos na agenda de políticas públicas.

\section{Respostas não-governamentais aos defeitos congênitos}

\section{- Estudo Colaborativo Latino-Americano de Malformações Congênitas (ECLAMC)}

O ECLAMC existe desde 1967 e é reconhecido como centro colaborador da Organização Mundial da Saúde (OMS) para a prevenção das malformações congênitas. Trata-se de um estudo epidemiológico voluntário, de base hospitalar, que congrega profissionais dedicados ao estudo das anomalias congênitas na América Latina. O espírito colaborativo, guiado por uma regulamentação operacional, assegura a uniformidade das avaliações, necessárias para a comparabilidade dos dados registrados nos vários centros 16.

O programa é dedicado à investigação dos defeitos congênitos, suas freqüências e fatores de risco, com o objetivo de preveni-los, promovendo as ações de saúde pertinentes 17. Segundo Salzano 18, trata-se do programa de monitoramento das malformações congênitas mais importante em nosso meio.

\section{- Sistemas de Informação sobre Agentes Teratogênicos (SIATs)}

Define-se como agente teratogênico qualquer substância, organismo, agente físico ou estado de deficiência que, estando presente durante a vida embrionária ou fetal, produz uma alteração na estrutura ou função da descendência. Considerando a amplitude da bibliografia sobre teratogenicidade e necessidade constante de atualização, serviços especializados para fornecimento desse tipo de informação a médicos e pacientes foram criados a partir da década de 80 em países da Europa e América do Norte 19.

Em 1990, o SIAT (Sistema Nacional de Informação sobre Agentes Teratogênicos) foi implantado no Serviço de Genética Médica do Hospital de Clínicas de Porto Alegre (HCPA) da Universidade Federal do Rio Grande do Sul (UFRGS), tendo sido o primeiro desta natureza a operar na América Latina. Seus objetivos principais são a prevenção do aparecimento de defeitos congênitos na espécie humana decorrentes de exposições ambientais e o aprofundamento de conhecimentos sobre teratogênese em humanos. O serviço, destinado a gestantes e médicos de diversas especialidades, opera através de contato telefônico gratuito, fax ou internet, fornecendo informações sobre exposições de mulheres grávidas a agentes químicos, físicos e biológicos. O SIAT atua também na investigação da teratogenicidade de agentes ambientais, 
através do seguimento e observação do final de todas as gestações submetidas a consultas 19 .

Outros SIATs foram criados posteriormente no Brasil, estando atualmente em operação serviços semelhantes no Rio de Janeiro, São Paulo, Campinas e Salvador 7 .

\section{- Serviço de Informações sobre Erros Inatos do Metabolismo (SIEM)}

Essa iniciativa pioneira foi implantada em 2001, no HCPA/UFRGS, centro de referência nacional para erros inatos do metabolismo. O objetivo do serviço é apoiar médicos e profissionais de saúde em situações que envolvam suspeita clínica de erros metabólicos, patologias raras e pouco conhecidas, mesmo entre esses profissionais 20 .

O SIEM pode ser contactado através de ligação telefônica gratuita, sendo fornecidas orientações relativas à conduta clínica e laboratorial recomendadas para cada caso. O serviço disponibiliza informações para médicos de diversas especialidades em qualquer região do Brasil, contribuindo para o diagnóstico e instituição precoce de terapêutica.

\section{Respostas governamentais aos defeitos congênitos}

\section{- Programa Nacional de Triagem Neonatal (PNTN)}

A partir de 1990, começaram a surgir leis relacionadas à triagem neonatal 21,22, culminando com o PNTN em 2001 23,24. Tal programa foi iniciado com a triagem da fenilcetonúria e do hipotireoidismo congênito, doenças que levam a retardo mental grave quando não tratadas precocemente, e cujos diagnósticos e instituição precoce de tratamento permitem o desenvolvimento adequado da criança afetada.

O PNTN considera que as doenças a serem triadas devem seguir como critérios o fato de não apresentarem manifestações clínicas precoces, permitirem a detecção precoce por meio de testes seguros e confiáveis, serem amenizáveis mediante tratamento, serem passíveis de administração em programas com logística definida e terem uma relação custo benefício economicamente viável e socialmente aceitável 23,24. Ao ser instituído o PNTN no âmbito do Sistema Único de Saúde (SUS), foi recomendada a triagem para as seguintes doenças: fenilcetonúria (PKU), hipotireoidismo congênito (HC), doença falciforme e outras hemoglobinopatias (DF) e fibrose cística (FC), de acordo com as fases de implantação (Fase I: PKU e HC; Fase II: PKU, HC e DF; Fase III: PKU, HC, DF e
FC). Os serviços de referência seriam cadastrados como "Referência em Triagem Neonatal / Acompanhamento e Tratamento de Doenças Congênitas", devendo garantir a realização da triagem, confirmação diagnóstica e adequado acompanhamento e tratamento dos pacientes triados.

As experiências dos programas de triagem neonatal mostram dados importantes, como a possibilidade de cobertura com qualidade e agilidade, com integração entre rede básica e centro de referência 25 (Botler J. Comunicação pessoal; 2002). Como regra primordial, a triagem neonatal deveria estar baseada em laboratórios regionais de referência (não necessariamente em todos os estados da união) com supervisão estadual (ou regional) integrada à rede primária e com suporte clínico de centros de referência; a supervisão global deveria ficar sob responsabilidade do grupo técnico de assessoramento em triagem neonatal, instituído pela Secretaria de Assistência à Saúde 24. É de interesse não apenas epidemiológico, mas também econômico para o país, analisar os resultados dos programas nas diferentes regiões brasileiras, priorizando as doenças com real indicação para rastreamento segundo regionalizações étnicas, sem vinculação aos interesses econômicos ou privados para a ampliação indiscriminada dos testes 26 . O seguimento desse modelo permitiria a otimização da triagem neonatal, com detecção precoce dos afetados e instituição eficaz de terapêutica, resultando em ações efetivas para a prevenção de complicações clínicas e do retardo mental.

\section{- Programas especiais do Ministério da Saúde relacionados ao tratamento de doenças genéticas}

Tratamentos de algumas doenças genéticas vêm sendo assumidos pelo MS, regulamentados através de portarias especiais. O processo não é simples, sobretudo por questões relacionadas ao elevadíssimo custo de medicamentos, muitas vezes, envolvendo ações judiciais ou fortes “lobbies" por parte das famílias, justificados pela Constituição Federal, segundo a qual todos têm direito à saúde, a ser provida pelo Estado.

Há diversos argumentos contrários ao governo assumir certos tratamentos, principalmente pela carência de recursos da União e ações básicas de saúde que poderiam ser implementadas no lugar de tratar apenas um paciente com doença rara. Caso utilizada a unidade "anos de vida ajustados por incapacida$d e$ " (AVAI), não seria justificado aos países em desenvolvimento o investimento em ações de 
saúde com alto custo por AVAI ganho 27. Como argumento favorável, pode ser utilizada a "regra de resgate" (rule of rescue - Jonsen), na qual não se deve abandonar uma pessoa à própria sorte pelo alto custo de seu tratamento, se o mesmo está disponível e é comprovadamente eficaz 28. A opção pelo não tratamento também seria incompatível com o princípio da integralidade, assim como com os princípios constitucionais da universalidade e igualdade 27 .

São descritos abaixo dois programas do MS para tratamento de doenças genéticas:

\section{a) Programa de Osteogênese Imperfeita}

A osteogênese imperfeita é uma doença causada por mutações no colágeno tipo I, caracterizada por fragilidade óssea e osteopenia. Os pacientes apresentam múltiplas fraturas que podem evoluir com graves deformidades ósseas e limitação funcional. O tratamento com substâncias do grupo dos bifosfonatos leva à diminuição da reabsorção óssea e ganho de massa óssea, diminuição de fraturas e melhora na qualidade de vida.

Em dezembro de 2001, foi publicada portaria 29 autorizando o tratamento da osteogênese imperfeita no âmbito do SUS. O IFF/FIOCRUZ, centro de referência no Rio de Janeiro, iniciou, em abril de 2002, o tratamento dos portadores da osteogênese imperfeita com Pamidronato Dissódico, tendo quarenta pacientes sido inseridos nesse programa até maio de 2003 30. Seguindo-se as normas estabelecidas pela portaria, outros centros poderão ser cadastrados, estendendo o atendimento ao maior número possível de afetados em todas as regiões brasileiras.

\section{b) Programa de Doença de Gaucher}

A doença de Gaucher é uma doença metabólica hereditária, que compromete o metabolismo dos lipídeos; suas principais manifestações são o aumento do fígado e baço, alterações hematológicas e lesões ósseas. O tratamento de reposição enzimática foi introduzido na prática clínica na década de 1990.

No Brasil, a Associação Brasileira de Portadores da Doença de Gaucher foi fundada em 1994, tendo como objetivos a divulgação da patologia e a obtenção do tratamento pelo SUS para os afetados. O trajeto inicial foi bastante tortuoso, incluindo liminares e mandatos judiciais para a compra do medicamento a partir de 1995. Atualmente, a medicação é diretamente custeada pelo MS, tendo sido, em 2002, estabelecidos protocolo oficial para tratamento da doença e centros de referência estaduais para avaliação, tratamento e acompanhamento dos pacientes 31 . Atualmente, a Imiglucerase é a droga mais cara do programa de medicamentos extraordinários. Tal financiamento, contudo, tem possibilitado uma vida normal e produtiva para os brasileiros acometidos por essa doença rara.

Alguns pontos devem ser ressaltados a respeito dos programas terapêuticos para doenças genéticas disponíveis via SUS. Tratamentos para outros tipos de doenças metabólicas vêm sendo desenvolvidos, muito provavelmente estarão disponíveis em breve e virão a ser requisitados pelas famílias e associações de pacientes. É fundamental que novos programas sigam protocolos bem estabelecidos, a exemplo dos disponíveis atualmente. Aliadas às questões terapêuticas, deverão ser traçadas estratégias para prevenção de novos casos, sendo essencial o papel da genética clínica com o aconselhamento genético, considerando ser essa uma das poucas possibilidades de estabilização dos custos crescentes dos tratamentos 32 .

\section{- Ações governamentais relacionadas à prevenção e monitorização dos defeitos congênitos}

Além da triagem neonatal e tratamentos já descritos, três iniciativas recentes, caso efetivamente implementadas, poderão auxiliar sobremaneira a programação do atendimento e prevenção dos defeitos congênitos.

\section{a) Fortificação da farinha com ácido fólico}

Os defeitos de fechamento do tubo neural são malformações de etiologia multifatorial, com fatores genéticos e ambientais envolvidos na sua gênese. Já está bem estabelecida a relação entre a deficiência do ácido fólico, metabolismo materno da homocisteína e ocorrência de malformações desse grupo. Estudos controlados em países com prevalência aumentada de defeitos de fechamento do tubo neural mostraram que a suplementação dietética com folato em mulheres pouco antes e durante a fase inicial da gestação diminui esses riscos de ocorrência ${ }^{33}$. Trabalho realizado no Chile 34 mostrou redução de $31,0 \%$ na incidência de defeitos de fechamento do tubo neural após fortificação da farinha de trigo com $2,2 \mathrm{mg} / \mathrm{kg}$ de ácido fólico.

A Agência Nacional de Vigilância Sanitária (ANVISA), considerando as recomendações da OMS e da Organização Pan-Americana da Saúde (OPAS) quanto à fortificação de produtos alimentícios com ferro e ácido fólico, regulamentou tal fortificação no Brasil 35. A partir de 
junho de 2004, tornou-se obrigatória a fortificação das farinhas de trigo e milho com 150mcg/ $100 \mathrm{~g}$ de ácido fólico. Assim é possível que haja uma diminuição da incidência de defeitos de fechamento do tubo neural no Brasil, embora talvez a fortificação das farinhas apenas não seja suficiente, considerando, além das dimensões continentais do país, as diversidades dos hábitos alimentares nas regiões. Estudos epidemiológicos para aferir a real eficácia da medida adotada deverão ser programados.

\section{b) Programa Nacional de Imunizações}

O vírus da rubéola é altamente teratogênico, podendo levar a óbito intra-uterino e malformações fetais, sendo a prevenção da síndrome da rubéola congênita a principal finalidade da vacinação contra rubéola 36 . A partir da segunda metade da década de 1990, a vacina MMR (anti-sarampo, caxumba e rubéola) foi introduzida na faixa pediátrica no Brasil, deslocando, no entanto, o grupo mais acometido pela rubéola de escolares/adolescentes para 20-29 anos 37, aumentando os riscos de infecção em gestantes e síndrome da rubéola congênita. Atualmente, além da infância, é preconizada a vacinação seletiva de adolescentes do sexo feminino e de mulheres até 49 anos no pós-parto ou pós-aborto imediato 36. Campanhas de vacinação contra rubéola em mulheres vêm ocorrendo desde 2001 no país. A introdução da vacina é uma medida bastante eficaz, embora a vigilância epidemiológica permanente seja fundamental para o controle e erradicação da síndrome da rubéola congênita.

\section{c) Campo 34 da declaração de nascimento}

A partir de janeiro de 2000, a Declaração de Recém-nascido Vivo (DN) - documento oficial emitido pelas maternidades, sem o qual os pais não podem realizar o registro civil - conta com um novo campo de preenchimento, o Campo 34 38. Tal item permite que sejam registradas as anomalias congênitas apresentadas ao nascimento de forma sistemática.

Informação de teor semelhante àquela possível de obter-se com o Campo 34 inexiste na maioria dos países, e tais dados podem vir a ser de grande valia para a monitorização e prevenção dos defeitos congênitos no Brasil. O campo não é de preenchimento obrigatório, embora conte com a questão presença ou não de malformação congênita juntamente com espaço para sua descrição e classificação da anomalia segundo o CID-10 6, possibilitando um registro bastante completo dessas alterações.
Levantamento realizado na cidade de Porto Alegre, cruzando dados do Sistema de Nascidos Vivos com óbitos cuja causa básica foi uma malformação congênita, registrados no Sistema de Informação de Mortalidade, mostrou um sub-registro de $60,7 \%$ para as malformações congênitas na declaração de nascimento 39. Esse dado é fundamental para alertar para a subutilização do Campo 34; sua introdução apenas será relevante para programas relacionados aos defeitos congênitos caso seja implementado de forma efetiva, com o reconhecimento da importância do dado. Na cidade de Porto Alegre, o interesse da municipalidade, aliado a um serviço de referência em genética clínica com experiência em monitoramento de defeitos congênitos, fez surgir um programa de atendimento a crianças com defeitos congênitos 40 . Programas semelhantes poderão vir a ser desenvolvidos em todo o país, aliados a um registro nacional. A implementação efetiva do Campo 34 permitirá não apenas o conhecimento da realidade brasileira relacionada aos defeitos congênitos, como uma melhor programação de ações de saúde para atendimento e prevenção.

\section{Considerações finais}

Uma política visando às anomalias congênitas é urgente, conforme já demonstrado pela sua crescente importância. É evidente que as enfermidades genéticas e os defeitos congênitos incidem significativamente na saúde de nossa população, acentuando-se, ainda mais, à medida que o desenvolvimento sócio-econômico e sanitário favorecer a diminuição das enfermidades infecciosas e nutricionais 2 .

Segundo a OMS 32, para o melhor atendimento aos defeitos congênitos, deve haver uma modificação no padrão dos serviços de saúde necessários para o atendimento da população, desviando o foco de doenças agudas para o manejo de problemas crônicos e de programas verticais para sistemas integrados de atenção primária. Tal modificação passa a ser especialmente necessária quando a queda da mortalidade infantil atinge patamar abaixo de 40-50/ 1.000. Com tais cifras, um número significativo de crianças com condições potencialmente incapacitantes, que morreriam sem diagnóstico, não apenas sobrevivem, como passam a ser diagnosticadas e a necessitar de intervenções médicas e de suporte ao longo da vida. Segundo o Instituto Brasileiro de Geografia e Estatística (IBGE. Tábua Completa de Mortalidade-Ambos os Sexos - 2001 - Brasil. http://www.ibge. 
gov.br/home/estatistica/populacao/tabuadevida/textoambossexos2001.shtm, acessado em 09/Out/2003), a mortalidade infantil no Brasil, em 2001, foi de 32,6/1.000, já indicando a necessidade de introdução na agenda de um enfoque específico para a atenção, suporte, tratamento e prevenção dos defeitos congênitos.

Apesar do enorme progresso registrado durante a última década nos métodos de diagnóstico, prevenção e tratamento das enfermidades genéticas, uma abordagem racional para a questão dos defeitos congênitos dependeria menos de tecnologia e mais de planejamento. Ao analisar o sistema político de saúde no país, é possível observar, de forma absolutamente clara, o não reconhecimento "oficial" da problemática dos defeitos congênitos frente à insuficiência da resposta governamental à questão. Embora os dados estejam facilmente disponíveis nas bases de dados da saúde, é possível que os mesmos ainda não tenham vindo à tona devido a não sensibilização específica do corpo técnico do MS. Ações, no Brasil, principalmente no sentido da organização do atendimento inicial, diagnóstico, exames complementares, estratégias de tratamento disponíveis e estabelecimento de rotinas de acompanhamento a longo prazo são recomendadas. Também é fundamental ser mencionado o papel da prevenção, através de campanhas educativas e da ampliação do acesso ao aconselhamento genético. Finalmente, porém não menos importante, a educação e a sensibilização não apenas do público, como também dos profissionais de saúde aos defeitos congênitos deverão ser estimuladas.

Conforme já mostrado, o processo de entrada na agenda da questão dos defeitos congênitos vem ocorrendo de forma lenta e tímida. Embora algumas ações já tenham sido bem implementadas, um ponto crucial para a abordagem da questão como um todo não vem sendo contemplado: a organização da rede de serviços em genética. Na América Latina, desde a década de 70, foram criados serviços de genética isolados, em geral como parte de estabelecimentos hospitalares ou associados a escolas de medicina e institutos de pesquisa. Esses centros desenvolvem ações de diagnóstico e assessoramento genético, particularmente no campo das alterações cromossômicas, malformações congênitas e retardamento mental. Embora a modalidade operacional desses centros varie muito e dependa de circunstâncias locais, quase sempre os serviços prestados são incompletos, desvinculados dos programas gerais de assistência médica e baseados somente na demanda espontânea ou derivada dos poucos profissionais de saúde que sabem de sua exis- tência. Por esses motivos, seu grau de utilização pela população é reduzido e ainda não tiveram impacto significativo na política de saúde dos respectivos países 2 . Nesse sentido, configurar, no sistema de saúde, uma rede para o atendimento aos defeitos congênitos não deve ser considerado um luxo ou um despropósito, muito menos uma utopia.

As ações de saúde em matéria de genética não diferem conceitualmente do resto das ações de saúde e compreendem os diversos aspectos de diagnóstico, prevenção e tratamento das enfermidades de origem genética e dos defeitos congênitos. Os objetivos visados são: prevenção da ocorrência, atendimento dos problemas uma vez ocorridos (detecção e tratamento precoce), prevenção das complicações e minimização do dano (reabilitação médica, psicológica e social) 41. É, no entanto, indispensável que esses programas não sejam concebidos isoladamente, mas sim estreitamente integrados aos demais programas de saúde 41 .

No Brasil, embora a maioria dos serviços continue atuando de forma individual, alguns já estão mais integrados com a saúde comunitária. Trabalhos recentes têm deixado um pouco de lado os assuntos mais técnicos, concentrando-se na discussão de como a genética clínica pode trazer melhorias para a vida da comunidade. Faria et al. 42 e Brunoni 43 reforçam a importância do reconhecimento e inserção de fato da especialidade no SUS, contemplando a formação de grupo de trabalho com suporte governamental, visando traçar recomendações para a implementação e melhoria dos serviços de genética clínica e médica no país.

Visando à elaboração de programa de genética clínica no Brasil, é fundamental que haja um levantamento o mais completo possível sobre as ações já existentes relacionadas aos defeitos congênitos, conforme apresentado neste artigo, além de delinear o perfil dos serviços já estabelecidos no país. Compilação de informações sobre os serviços e propostas para integração da abordagem aos defeitos congênitos no âmbito do SUS foram recentemente apresentadas 7. Deve-se considerar que esses centros já estruturados deveriam formar a coluna vertebral de qualquer plano organizado que decida pôr em prática medidas de diagnóstico, prevenção e tratamento das enfermidades genéticas no âmbito comunitário ${ }^{2}$. A configuração em rede (regionalizada, hierarquizada e funcional, conforme preconizado pelo SUS) dos serviços em genética clínica seria um item crucial para a abordagem e atendimento aos defeitos congênitos de maneira integral, resolutiva e, sobretudo, democrática. 


\section{Resumo}

O impacto dos defeitos congênitos no Brasil vem aumentando progressivamente, tendo passado da quinta para a segunda causa dos óbitos em menores de um ano entre 1980 e 2000, apontando para a necessidade de estratégias específicas na política de saúde. Foram localizadas, no Brasil, direcionadas aos defeitos congênitos, ações governamentais e não-governamentais. Estas envolvem serviços de informação sobre agentes teratogênicos na gravidez e sobre doenças metabólicas geneticamente determinadas, monitorização de defeitos congênitos, programa de triagem neonatal e tratamento de algumas doenças genéticas, imunização contra rubéola, além da fortificação de farinhas com ácido fólico como ação preventiva de certos defeitos congênitos. Apesar da importância de tais iniciativas, é pouco provável que seja possível atender à questão dos defeitos congênitos de forma integrada. Para a efetivação de um sistema de atenção voltado aos defeitos congênitos, deverá ser formulada política específica, de âmbito nacional, com a participação ativa do Ministério da Saúde, utilizando, como espinha dorsal, os serviços de genética existentes. Só assim, será possível a estruturação de uma rede regionalizada, hierarquizada e funcional voltada à atenção aos defeitos congênitos no Brasil.

Defeitos Congênitos; Política de Saúde; Serviços em Genética; Genética Médica; Planejamento em Saúde

\section{Colaboradores}

D. D. G. Horovitz contribuiu na pesquisa, elaboração e redação do artigo. J. C. Llerena Jr. e R. A. Mattos colaboraram na orientação e revisão do manuscrito.

\section{Referências}

1. Lynberg MC, Edmonds LD. State use of birth defects surveillance. In: Wilcox LS, Marks JS, editors. From data to action. CDC's public health surveillance for women, infants and children. Atlanta: U.S. Department of Health \& Human Services, Public Health Service, Centers for Disease Control and Prevention; 1995. p. 217-29.

2. Organização Pan-Americana da Saúde. Prevenção e controle de enfermidades genéticas e os defeitos congênitos: relatório de um grupo de consulta. Washington DC: Organização Pan-Americana da Saúde; 1984. (Publicação Científica 460).

3. Penchaszadeh VB, Christianson AL, Giugliani R, Boulyjenkov V, Katz M. Services for the prevention and management of genetic disorders and birth defects in developing countries. Community Genet 1999; 2:196-201.

4. Nussbaum RL, McInnes RR, Willard HF. Introduction - classification of genetic disorders. In: Thompson \& Thompson Genetics in Medicine. 6th Ed. Philadelphia: Saunders; 2001. p. 2-3.

5. Bardin L. Análise de conteúdo. Lisboa: Edições 70; 1979.

6. Organização Mundial da Saúde/Centro Colaborador da OMS para a Classificação de Doenças em Português, Universidade de São Paulo. Classificação estatística internacional de doenças e problemas relacionados à saúde - CID-10. São Paulo: Edusp; 1994.

7. Horovitz DDG. Atenção aos defeitos congênitos no Brasil: propostas para estruturação e integração da abordagem no sistema de saúde [Tese de Doutorado]. Rio de Janeiro: Instituto de Medicina Social, Universidade do Estado do Rio de Janeiro; 2003.

8. Nazer J, Castillo S, Cifuentes L, Ruiz G, Pizzaro MT, Parada L. Incidence of congenital malformations in Chile from 1969 to 1986. Results of a LatinAmerican collaborative study. Rev Med Chil 1989; 117:219-27.

9. Powell-Griner E, Woolbright A. Trends in infant deaths from congenital anomalies: results from England and Wales, Scotland, Sweden and the United States. Int J Epidemiol 1990; 19:391-8.

10. Wertz DC, Fletcher JC, Berg K, Boulyjenkow V. Guidelines on ethical issues in medical genetics and the provision of genetics services. Geneva: World Health Organization; 1995.

11. Wertz DC, Fletcher JC, Berg K. Review of ethical issues in medical genetics. Geneva: World Health Organization; 2001.

12. Instituto Fernandes Figueira, Fundação Oswaldo Cruz. Boletim de produção de serviços. Arquivo nosológico gerado em 28/Ago/2003. Rio de Janeiro: Departamento de Informação e Documentação, Instituto Fernandes Figueira, Fundação Oswaldo Cruz; 2003.

13. Paskulin GA. Casuística e caracterização das patologias hereditárias em um hospital pediátrico [Dissertação de Mestrado]. Porto Alegre: Universidade Federal do Rio Grande do Sul; 1989.

14. Brunoni D. Estado atual do desenvolvimento dos serviços de genética médica no Brasil. Rev Bras Genet 1997; 20 Suppl:11-23. 
15. Winter RM, Knowles SAS, Bieber FR, Baraitser M. The malformed fetus and stillbirth - a diagnostic approach. Chichester: John Wiley \& Sons; 1989.

16. Castilla EE, Orioli IM. ECLAMC: The Latin-American Collaborative Study of Congenital Malformations. Community Genet 2004; 7:76-94.

17. Castilla EE, Lopez-Camelo JS, Paz JE, Orioli IM. Introducción. In: Dutra MG, organizador. Prevención primaria de los defectos congénitos. Rio de Janeiro: Editora Fiocruz; 1996. p. 11-7.

18. Salzano FM. Saúde pública no primeiro e terceiro mundos: desafios e perspectivas. Ciênc Saúde Coletiva 2002; 7:7-16.

19. Schüller-Faccini L, Schvartzman L, Cecchin C. Teratogênese humana e o SIAT. In: Sanseverino MT, Spritzer D, Schüller-Faccini L, organizadores. Manual de teratogênese. Porto Alegre: Editora da Universidade; 2001. p. 11-7.

20. Brustolin S, Souza C, Pires R, Refosco L, Giugliani R. Serviço de informações sobre erros inatos do metabolismo (SIEM - 08005102858). In: Anais do XV Congresso Brasileiro de Genética Clínica. Porto Alegre: Sociedade Brasileira de Genética Clínica; 2003. p. 117.

21. Brasil. Lei n. 8.069 de 13 de julho de 1990. Dispõe sobre o Estatuto da Criança e do Adolescente e dá outras providências. Inciso III, artigo 10. Diário Oficial da União 1990; 16 jul.

22. Brasil. Portaria GM/MS n. 22 de 15 de janeiro de 1992. Trata do programa de diagnóstico precoce do hipotireoidismo congênito e fenilcetonúria Brasília: Gabinete do Ministro, Ministério da Saúde.

23. Brasil. Portaria GM/MS n. 822 de 6 de junho de 2001. Institui, no âmbito do Sistema Único de Saúde, o Programa Nacional de Triagem Neonatal / PNTN. Brasília: Gabinete do Ministro, Ministério da Saúde.

24. Brasil. Portaria SAS n. 189 de 6 de junho de 2001. Institui, no âmbito da Secretaria de Assistência à Saúde - Ministério da Saúde, o Grupo Técnico de Assessoramento em Triagem Neonatal. Brasília: Secretaria de Assistência à Saúde, Ministério da Saúde.

25. Aguiar MJB. Experiências dos programas de triagem neonatal. In: Anais do XIV Congresso Brasileiro de Genética Clínica. Ribeirão Preto: Sociedade Brasileira de Genética Clínica; 2002. p. 16.

26. Llerena Jr. JC. Genética médica, Sistema Único de Saúde brasileiro (SUS) e integralidade na atenção e no cuidado à saúde. Ciênc Saúde Coletiva 2002; $7: 21-5$.

27. Mattos RA. Integralidade e a formulação de políticas específicas de saúde. In: Pinheiro R, Mattos RA, organizadores. Construção da integralidade: cotidiano, saberes e práticas em saúde. Rio de Janeiro: Instituto de Medicina Social, Universidade do Estado do Rio de Janeiro/ABRASCO; 2003. p. 45-59.

28. Clarke J, Amato D, Deber R. Managing public payment for high-cost, high-benefit treatment: enzyme replacement therapy for Gaucher's disease in Ontario. CMAJ 2001; 165:595-6.

29. Brasil. Portaria GM/MS n. 2.305, de 19 de dezembro de 2001. Aprova o Protocolo de Indicação de Tratamento Clínico da osteogenesis imperfecta com pamidronato dissódico no âmbito do Sis- tema Único de Saúde - SUS. Brasília: Gabinete do Ministro, Ministério da Saúde; 2001.

30. Barbosa C, Zlot R, Horovitz D, Rodrigues MC, Llerena J. Experiência em 29 pacientes submetidos ao primeiro ciclo do pamidronato dissódico no centro de referência IFF/FIOCRUZ para osteogênese imperfeita no Rio de Janeiro. In: Anais do XV Congresso Brasileiro de Genética Clínica. Porto Alegre: Sociedade Brasileira de Genética Clínica; 2003. p. 19.

31. Brasil. Portaria SAS n. 449, de 8 de julho de 2002. Aprova o protocolo clínico e diretrizes terapêuticas - doença de Gaucher - Imiglucerase. Diário Oficial da União 2002; 9 jul.

32. World Health Organization. Primary health care approaches for prevention and control of congenital disorders and disability. Geneva: World Health Organization; 2000.

33. Nussbaum RL, McInnes RR, Willard HF. Genetics of disorders with complex inheritance - neural tube defects. In: Nussbaum RL, McInnes RR, Willard HF, editors. Thompson \& Thompson genetics in medicine. $6^{\text {th }}$ Ed. Philadelphia: Saunders; 2001. p. 304-6.

34. Castilla EE, Orioli IM, López-Camelo JS, Dutra MG, Nazer-Herrera J. Preliminary data on changes in neural tube prevalence rates after folic acid fortification in South America. Am J Med Genet 2003; 123:123-8.

35. Brasil. Resolução - RDC n. 344, de 13 de dezembro de 2002. Aprova o Regulamento Técnico para a Fortificação das Farinhas de Trigo e das Farinhas de Milho com Ferro e Ácido Fólico. Diário Oficial da União 2002; $18 \mathrm{dez}$.

36. Brasil. Manual de normas de vacinação. 3a Ed. Brasília: Fundação Nacional de Saúde, Ministério da Saúde; 2001.

37. Rymkiewicz E, Zuzarte FCC, Campos SO, Pinto MIM. Rubella immunization strategies in the state of São Paulo, Brazil. Bull World Health Organ 2002; 80:258.

38. Brasil. Sistema de informações sobre nascidos vivos. Manual de instruções para o preenchimento da declaração de nascido vivo. 3a Ed. Brasília: Ministério da Saúde; 1999.

39. Cunha J, Aerts D, Leite JCL, Castilla EE. Defeitos congênitos em Porto Alegre: uma investigação da qualidade dos dados registrados na Declaração de Nascido Vivo. Rev Bras Epidemiol 2002; 5 Suppl:51.

40. Schüller-Faccini L, Leite JCL, Sanseverino MTV, Peres RM. Avaliação de teratógenos potenciais na população brasileira. Ciênc Saúde Coletiva 2002; 7:65-71.

41. World Health Organization. Technical report series on the control of hereditary diseases. Geneva: World Health Organization; 1993.

42. Faria APM, Ferraz VEF, Acosta AX, Brunoni D. Clinical genetics in the developing countries: the Brazilian situation. Community Genet 2004; 7:95-105.

43. Brunoni D. Aconselhamento genético. Ciênc Saúde Coletiva 2002; 7:101-7.

Recebido em 10/Nov/2004

Versão final reapresentada em 24/Jan/2005

Aprovado em 31/Jan/2005 